\title{
Methane-specific gas detectors: the effect of natural gas composition.
}

\section{J Hodgkinson $^{\mathrm{a}}$ and R D Pride ${ }^{\mathrm{b}}$}

Advantica Ltd, Ashby Rd, Loughborough, Leics LE11 3GR, UK

a Now at: Engineering Photonics Group, School of Engineering, Cranfield University, Cranfield, Bedfordshire, MK43 0AL, UK.

b Now at: Institute for the Protection and Security of the Citizen (IPSC), Security and Reliability of Energy Supplies, Joint Research Centre, Ispra, VA 21020, Italy

j.hodgkinson@cranfield.ac.uk

\begin{abstract}
Certain gas sensors, particularly those based on optical spectroscopy, have enabled the detection of individual gas species such as methane with low cross-sensitivity to other gases. For gas-specific instruments used to measure natural gas leaks, this paper considers whether it is necessary to consider the other components of natural gas in addition to methane. We have quantified the effect of gas compositional variation on methane-based measurements on the ppm, \%LEL and \%volume scales. \%LEL measurements, important for safety applications, are the most challenging for methane-specific detection. Acceptable levels of error have been drawn from gas detector standards and by comparison with established gas detectors. The fundamental error expected from a methane-specific detector, as a result of variations in gas composition, would be larger than this benchmark on the \%LEL scale. However for gas-specific detection, measurement of an additional component such as ethane is shown to reduce the error to below the benchmark level. This has been demonstrated experimentally using an instrument based on tunable diode laser spectroscopy.
\end{abstract}


When responding to public reported gas escapes, many gas utilities currently use pellistor - based detectors and flame ionization detectors to measure the gas concentration. This concentration can be expressed in either parts per million (ppm), \% volume, or as a percentage of the lower explosive limit (LEL) of the gas mixture, the latter being an important safety parameter ${ }^{[1]}$. In traditional instruments, a different sensor technology is typically employed for each range. Thermal conductivity sensors are used to measure \%volume concentrations in these sensors. Pellistor sensors are used to detect gas concentrations on the \%LEL scale, and consist of a catalyst bead on which flammable gases are oxidized at high temperature ${ }^{[2]}$. This exothermic reaction raises the bead temperature still further and increases the bead's electrical resistance. High sensitivity flame ionization detectors (FIDs) are used on the ppm range and burn the gas directly in a hydrogen flame ${ }^{[3]}$. Because of their operating principles, both pellistors and FIDs provide a measure of concentration that is inherently related to the combustibility of the natural gas leak.

Natural gas consists predominantly of methane, and there has been great interest in optical sensors, based on measuring methane's infrared absorption, as a means of detecting and quantifying natural gas leaks. These developments have raised the issue of cross-sensitivity of instruments to the different components of natural gas. Narrow-band tunable laser diodes are available in the near infrared offering a high degree of spectral resolution that enables the detection of single gas components ${ }^{[4,5]}$. For natural gas applications, much activity has concentrated on methane detectors based on optical absorption around $1.65 \mu \mathrm{m}^{[6,7]}$, with no cross-sensitivity to other flammable gas components. Alternatively, non-dispersive infrared instruments can be used at $3.3 \mu \mathrm{m}^{[8]}$, with undefined crosssensitivity to the other gas components. If such cross-sensitivities are not considered properly, the resulting error can be large. Advantica has observed proportional errors as high as $60 \%$ in commercial instruments based on non-dispersive infra-red (NDIR) technology, that were calibrated using methane alone but used to measure natural gas ${ }^{[9]}$.

We have now analysed the possible effect of a methane-specific measurement on the validity of the resulting ppm, \%LEL and \%vol concentration estimates. The analysis is relevant to any gas-specific measurement and not limited to the optical techniques mentioned above. This paper seeks to establish whether these new developments would be fundamentally compatible with industry requirements, using the following approach:

(i) Establish benchmark levels of acceptable uncertainty for portable natural gas detectors, drawn from instrument standards and by comparison with the level of error in established instruments. 
(ii) Calculate the expected level of error for a hypothetical, ideal instrument that responds to methane alone. Data on the composition of natural gas in different geographical regions was taken from a variety of sources.

We repeated this analysis for a hypothetical instrument able to make simultaneous measurement of both methane and ethane. The approach was considered for two reasons. Firstly, after methane, ethane is the flammable component present in the greatest quantities and therefore it may be easier to detect than any of the other flammable components. Secondly, there are regions of the infrared spectrum that contain both individual methane and ethane absorption lines. Indeed a dual methane plus ethane measurement has been developed ${ }^{[10]}$, and we have tested this with a range of gas compositions to demonstrate the validity of the approach.

\section{The composition of natural gas}

If natural gas were composed of methane alone, or indeed if methane and the other components were present at levels that did not change, there would have been no need to do this work. However, their proportions are variable and it is necessary to understand the nature and extent of this variation in composition. Natural gas comprises mainly methane $\left(\mathrm{CH}_{4}\right)$, with a small proportion of higher hydrocarbons such as ethane $\left(\mathrm{C}_{2} \mathrm{H}_{6}\right)$, propane $\left(\mathrm{C}_{3} \mathrm{H}_{8}\right)$, butane $\left(\mathrm{C}_{4} \mathrm{H}_{10}\right)$ and so on. Inert gases such as nitrogen $\left(\mathrm{N}_{2}\right)$ and carbon dioxide $\left(\mathrm{CO}_{2}\right)$ are present at the level of a few per cent volume, and various compounds can be present in ppm quantities, including the odorant.

Portable gas detectors are used to detect leaks from gas distribution networks, in jobs that can last from around 30 minutes to an 8 hour shift, in a variety of unpredictable locations within one region or one organization's jurisdiction. It is impractical in most cases to have prior knowledge of local variations in gas composition, and in any case compositions in some networks can vary significantly

over the course of a few minutes ${ }^{[11]}$. Therefore, any one instrument will be required to cope with a wide range of gas compositions without any changes to its calibration settings.

Various sources of data are available to assess the degree of compositional variability in the gas distribution network. In the UK, a reliable source comes from the gas chromatographs (Danalyzer 500, Daniel Instruments, Houston, USA) operated by the UK gas transmission and distribution companies, which provide full information on gas composition from samples taken every four minutes. For this study, over 15,000 compositions recorded by these analysers at network entry points have been sampled from a period covering a complete calendar year (2000), so as to include the full range of potential seasonal or diurnal effects. Using entry point data gives the worst case for compositional 
extrema but cannot quantify any effects caused by subsequent downstream blending of gases from different supply points.

Other sources used in this study cover European networks, from a survey of GERG members (Group Européen de Recherches Gazières) ${ }^{[12]}$, a series of typical US compositions obtained from an American Gas Association (AGA) project ${ }^{[13]}$ and an ad hoc set of typical compositions from the rest of the world collected by Advantica over a period of several years (designated "RoW"). We have presented the results for different geographical regions separately in this paper. For the non-UK studies the data may represent typical average compositions for different sources and we are therefore uncertain as to the full possible extent of variations for instantaneous compositions. Nevertheless, experience with the UK data suggests that the difference in composition between different sources ${ }^{[14]}$ (such as different gas fields) tends to be greater than the range of compositions encountered from a single source.

Only those components considered significant were used for calculations, namely the levels of inert gases $\left(\mathrm{N}_{2}\right.$ and $\left.\mathrm{CO}_{2}\right)$ plus hydrocarbons from methane through to $\mathrm{C}_{6}$. Table 1 shows the chief flammable constituents of natural gas, their lower explosive limits and the composition range found at entry points to the UK gas transmission system.

Table 1. LELs and composition ranges for key flammable constituents of natural gas in the UK.

Reproduced from [14].

\begin{tabular}{|c|c|c|}
\hline Gas & $\underline{\text { Lower explosive limit in air }^{\mathrm{a}}}$ & $\underline{\text { Range in natural gas in the UK }}$ \\
\hline methane & $4.9 \% \mathrm{vol}$ & $85-95 \%$ \\
\hline ethane & $2.8 \% \mathrm{vol}$ & $2-9 \%$ \\
\hline propane & $2.0 \% \mathrm{vol}$ & $0.5-3 \%$ \\
\hline butanes & $1.5 \% \mathrm{vol}$ & $0.1-0.4 \%$ \\
\hline pentanes & $1.4 \% \mathrm{vol}$ & $0.001-0.5 \%$ \\
\hline
\end{tabular}

a LEL values in general use within the natural gas industry, as stated by the Institute of Gas Engineers and Managers ${ }^{[1]}$. Note however that IEC 60079-20 $0^{[15]}$ defines LELs of many hydrocarbons differently, for example the LEL for methane is stated to be $4.4 \%$ vol and LELs of many other hydrocarbons are also reduced. This change would not significantly alter the conclusions of this study, because we are concerned with proportional and relative changes or errors in the LELs of different gas mixtures. 
There are two points to note from Table 1. Firstly, the range of concentrations of the non-methane hydrocarbons can be relatively large. Secondly, the LELs of the different constituents are significantly different, with flammability generally increasing with increased length of the carbon chain. Therefore, the LEL of the resulting mixture can vary, and the variation in the non-methane components could cause significant errors for a methane - specific \%LEL detector. These errors are quantified in section 5 .

After methane, ethane is typically the most significant flammable component, but the approach taken here could equally be applied to any other secondary component. The proportions of methane and ethane reported for the gas compositions used in this study are displayed graphically in Figure 1.

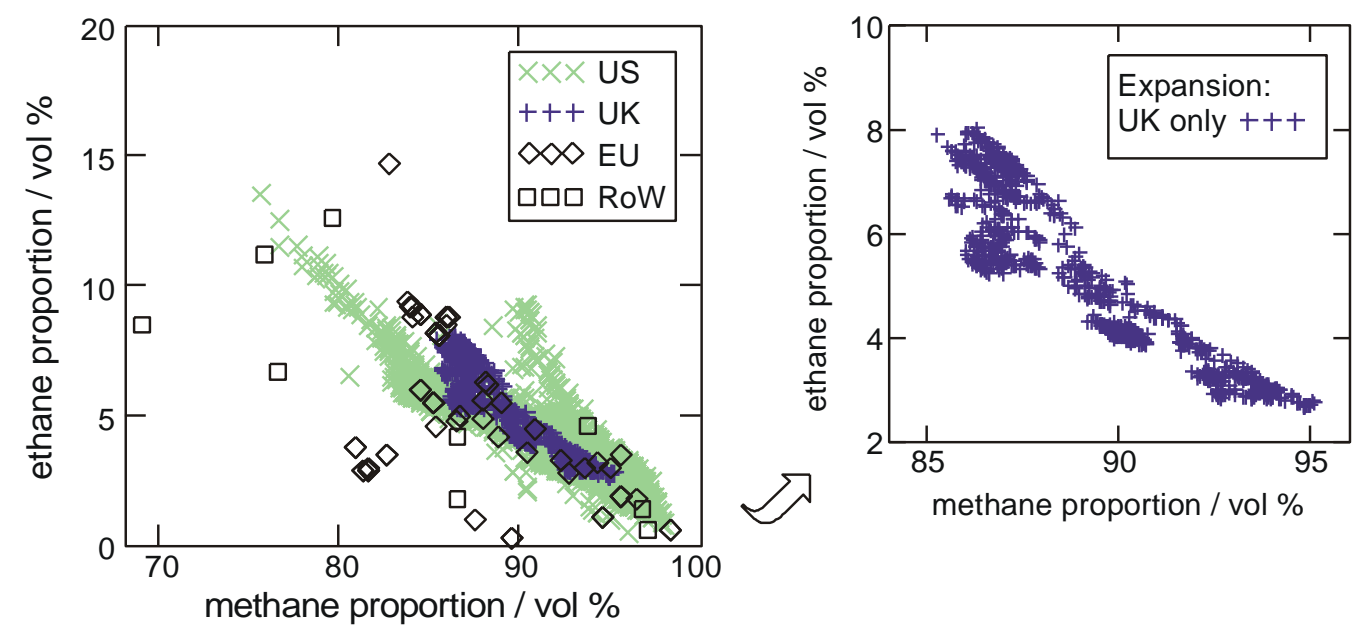

Figure 1. Proportions of methane and ethane for the gas compositions used in this study.

\subsection{LELs of natural gas mixtures}

In a mixture comprising different flammable and non-flammable components, the determination of the true LEL of the combined gas mixture is complex, especially when the mixture itself contains a proportion of inert gases such as $\mathrm{N}_{2}$ and $\mathrm{CO}_{2}$. The LEL arises not simply from the amount of energy that would be released on combustion, but also from a consideration of flame transport factors. In our work, the true LEL of each gas mixture was calculated using the method established by Coward and Jones ${ }^{[16]}$, which consists of the following steps.

1. The mixture is dissected into simpler component mixtures, each of which consists of only one flammable gas or only one flammable gas plus all or part of the inert gases. According to Coward and Jones, this dissection is "somewhat arbitrary", within certain well-defined limits. In practice 
for many natural gas mixtures, the inert gases would be allocated to a component mixture with methane, the largest single constituent, and / or ethane, the second.

2. The LELs of the simple mixtures containing inerts are then determined from charts given by Coward and Jones ${ }^{[16]}$.

3. The LEL of the overall mixture is then given by the equation

$$
L E L_{\text {mix }}=\frac{100 \%}{\frac{p_{1}}{N_{1}}+\frac{p_{2}}{N_{2}}+\frac{p_{3}}{N_{3}}+\cdots}
$$

Where $p_{1}, p_{2}, p_{3}$ etc are the proportions of the simple component mixtures, and $N_{1}, N_{2}, N_{3}$ etc are the respective LELs of these mixtures determined in step 2.

We have used the above method in this work to quickly determine the "true" LEL of a wide range of natural gas mixtures. The validity of the approach was examined by Jones in 1929 in a series of experiments to determine the true LEL of a variety of gas mixtures, which values were then compared with the results of the calculation above ${ }^{[17]}$. However, this study considered gas mixtures that were not representative of natural gases; in particular they contained significant quantities of inert gas $(50 \%-85 \%)$, which may be considered as a major source of uncertainty. For the 7 mixtures with under $75 \%$ inert content, agreement between calculated and measured values was between 0 and $8 \%$ of the experimental LEL. We consider the maximum error to be somewhat large compared to the instrumental requirement discussed here, albeit for uncharacteristic gas mixtures.

An approach used to support the Coward and Jones method is to calculate burning velocities for the different mixtures in an iterative combustion model, however this is computationally intensive. The method involves calculation of burning velocities using the Sandia Laboratories PREMIX code ${ }^{[18]}$, utilizing the validated GRI-Mech reaction mechanism ${ }^{[19]}$. The rationale is that if a flame cannot propagate (i.e. has a burning velocity close to zero) then it must be outside of the flammable range. For this work, repeated iterations were made with adjustment to the mixture composition of the unreacted gas starting at stoichiometric conditions and then progressing to leaner mixtures until the burning velocity was close to zero. A small extrapolation was then performed and the LEL defined as the value obtained from the intercept. Three extreme example mixtures were chosen and the resulting LELs compared with the results of the above method. Agreement was within $0.07 \%$ vol or $1.5 \%$ of the LEL value. How this might affect the results of our calculations then depends on whether this potential error is independent of, or correlated with, our calculated instrumental errors. In the latter (worst) case, the proportional error in our calculated figures could be up to $\pm 1.5 \%$, which is low compared to potential instrumental errors considered in this paper. 


\section{$3 \quad$ Standards for gas detectors}

We now need to establish the level of measurement error that might be acceptable for portable gas detectors. Sources of such information include international standards and the sometimes more stringent internal company standards; where organizations such as National Grid (NG) are concerned, such standards are made available to interested parties on request.

Standards considered in this study are compared in Table 2. Allowable errors vary through the gas concentration range and with environmental conditions. For the purpose of simplifying the comparison, one example set of conditions (concentration, temperature change, pressure change) has been chosen on each range.

Standard IEC / EN 60079 part $29^{[20]}$ covers performance requirements for flammable gas detectors on the \%LEL and \%vol ranges, and includes testing with "other gases" representative of those for which the apparatus is claimed to be suitable. This allows for testing with a range of natural gas mixtures. The errors allowed under IEC / EN 60079 for "other gases" have therefore been adopted here as benchmarks representative of worldwide gas detection applications.

In the UK, internal company standards maintained by National Grid are effectively adopted by the majority of the UK gas industry and have been used as the basis for UK-relevant benchmarks. For $\%$ LEL and \%volume measurements the relevant NG standard is INQ $3^{[21]}$ while the draft standard INQ4 ${ }^{[22]}$ relates to parts per million by volume (ppm) measurements, and these are historically related to the expected type test performance of two established instruments: respectively the GMI Gascoseeker (GMI, Gas Measurement Instruments Ltd, Renfrew, Scotland) and the Telegan Gas-Tec FID (Telegan Gas Detection, Abingdon, UK). As there is no explicit consideration of "other gases" here, we have instead taken the allowable error in response to pressure variation as a benchmark, this being another uncontrolled variable. 
Table 2 (a). Allowable error levels for detectors on the \%vol scale.

\begin{tabular}{|c|c|c|c|c|c|}
\hline \multirow[b]{2}{*}{$\underline{\text { Effect }}$} & \multirow[b]{2}{*}{ Example condition } & \multicolumn{2}{|c|}{$\underline{\text { IEC / EN 60079-29 }}{ }^{[15]}$} & \multicolumn{2}{|c|}{$\underline{\mathrm{NG} \text { INQ3 }}{ }^{[21]}$} \\
\hline & & Error & $\frac{\text { Proportional }}{\text { error }}$ & Error & $\frac{\text { Proportional }}{\text { error }}$ \\
\hline Calibration curve & $50 \%$ vol & $\pm 5 \%$ vol & $\pm 10 \%$ & $\pm 2 \% \mathrm{vol}$ & $\pm 4 \%$ \\
\hline Temperature & $-10^{\circ} \mathrm{C}$ (change from $20^{\circ} \mathrm{C}$ ) & $\pm 7 \%$ vol & $\pm 14 \%$ & $\pm 5 \%$ vol & $\pm 10 \%$ \\
\hline Pressure & $80 \mathrm{kPa}$ (change from $100 \mathrm{kPa}$ ) & $\pm 15 \%$ vol & $\pm 30 \%$ & $\pm 3 \%$ vol & $\pm 6 \% *$ \\
\hline "Other gases" & $50 \%$ vol & $\pm 7.5 \% \mathrm{vol}$ & $\pm 15 \% *$ & \multicolumn{2}{|c|}{ none specified } \\
\hline
\end{tabular}

Table 2 (b). Allowable error levels for detectors on the \%LEL scale.

\begin{tabular}{|c|c|c|c|c|c|}
\hline \multirow[b]{2}{*}{$\underline{\text { Effect }}$} & \multirow[b]{2}{*}{ Example condition } & \multicolumn{2}{|c|}{ IEC / EN $60079^{[15]}$} & \multicolumn{2}{|c|}{ NG INQ3 ${ }^{[21]}$} \\
\hline & & Error & $\begin{array}{c}\text { Proportional } \\
\text { error }\end{array}$ & Error & $\begin{array}{l}\text { Proportional } \\
\text { error }\end{array}$ \\
\hline Calibration curve & $50 \%$ LEL & $\pm 5 \% \mathrm{LEL}$ & $\pm 10 \%$ & $\pm 3 \% \mathrm{LEL}$ & $\pm 6 \% *$ \\
\hline Temperature & $-10^{\circ} \mathrm{C}$ (change from $20^{\circ} \mathrm{C}$ ) & $\pm 5 \%$ LEL & $\pm 10 \%$ & $\pm 5 \% \mathrm{LEL}$ & $\pm 10 \%$ \\
\hline Pressure & $80 \mathrm{kPa}$ (change from $100 \mathrm{kPa}$ ) & $\pm 15 \%$ LEL & $\pm 30 \%$ & $\pm 3 \%$ LEL & $\pm 6 \% *$ \\
\hline "Other gases" & $50 \%$ LEL & $\pm 7.5 \% \mathrm{LEL}$ & $\pm 15 \% *$ & \multicolumn{2}{|c|}{ none specified } \\
\hline
\end{tabular}

Table 2 (c). Allowable error levels for detectors on the ppm scale.

\begin{tabular}{|c|c|c|c|c|c|}
\hline \multirow[b]{2}{*}{$\underline{\text { Effect }}$} & \multirow[b]{2}{*}{ Example condition } & \multicolumn{2}{|c|}{$\underline{\text { IEC / EN 60079-29 }}{ }^{[15]}$} & \multicolumn{2}{|c|}{$\underline{\mathrm{NG} \text { INQ3 }}{ }^{[21]}$} \\
\hline & & Error & $\frac{\text { Proportional }}{\underline{\text { error }}}$ & $\underline{\text { Error }}$ & $\frac{\text { Proportional }}{\text { error }}$ \\
\hline Calibration curve & $500 \mathrm{ppm}$ & \multicolumn{2}{|c|}{ none specified } & $\pm 75 \mathrm{ppm}$ & $\pm 15 \%$ \\
\hline $\begin{array}{l}\text { Temperature and } \\
\text { pressure combined }\end{array}$ & $\begin{array}{l}-5^{\circ} \mathrm{C} \text { to }+40^{\circ} \mathrm{C} \text { and } \\
900-1050 \mathrm{mbar}\end{array}$ & \multicolumn{2}{|c|}{ none specified } & $\pm 125 \mathrm{ppm}$ & $\pm 25 \% *$ \\
\hline "Other gases" & 500 ppm & \multicolumn{2}{|c|}{ none specified } & \multicolumn{2}{|c|}{ none specified } \\
\hline
\end{tabular}

* Highlighted allowable errors have been selected for later benchmarking in this paper.

Stringent error limits apply on the \%vol and \%LEL scales, since the results of these measurements can be important safety criteria. Lower ( $\mathrm{ppm}$ ) concentrations of natural gas are typically used (a) to confirm the absence or presence of a gas leak, and (b) to locate the source. The first requirement demands a reliable and accurate zero, the second requirement demands high sensitivity and sufficient accuracy to enable areas of higher and lower gas concentration to be identified. The ppm application is therefore less demanding in terms of scale accuracy than for \%LEL and \%vol readings. 


\section{$4 \quad$ Performance of existing instruments}

\subsection{Gas test mixtures}

A series of gas test mixtures was chosen by reference to the UK composition data described in section 2. Mixtures were chosen from a range of possible compositions to give worst case test conditions for instruments, having a wide range of compositional variation within a small number of test samples, as shown in Table 3. They represent a worst test case for UK instruments with crossresponse to methane and ethane, having extremes of proportions of these components while excluding outliers. Mixtures A, B and D were chosen for their extremes of composition, while mixture $\mathrm{C}$ was chosen as a median, central representative so as not to neglect the mainstream.

Table 3. Compositions of different test gas mixtures used in this study, with their LELs calculated according to the method of Coward and Jones ${ }^{[16]}$.

\begin{tabular}{|l|l|l|l|l|}
\hline Component & \multicolumn{4}{|l}{ Composition / \%ol } \\
\hline & Mixture A & Mixture B & Mixture C & Mixture D \\
\hline nitrogen & 1.3 & 0.84 & 2.3 & 4.72 \\
\hline carbon dioxide & 0.346 & 2.54 & 1.06 & 1.22 \\
\hline methane & 94.87 & 85.71 & 90.14 & 86.34 \\
\hline ethane & 2.71 & 7.63 & 4.61 & 5.42 \\
\hline propane & 0.46 & 2.43 & 1.21 & 1.48 \\
\hline i-butane & 0.073 & 0.211 & 0.172 & 0.205 \\
\hline n-butane & 0.096 & 0.434 & 0.258 & 0.334 \\
\hline pentanes & 0.060 & 0.151 & 0.151 & 0.176 \\
\hline hexanes & 0.092 & 0.057 & 0.096 & 0.104 \\
\hline LEL / \%vol & $4.81 \pm 0.05$ & $4.53 \pm 0.05$ & $4.71 \pm 0.05$ & $4.77 \pm 0.05$ \\
\hline
\end{tabular}

The test compositions were selected from a list of real compositions, rather than using mean values. Figure 2 shows two representations of the range of UK compositions considered, with mixtures A-D superimposed. 


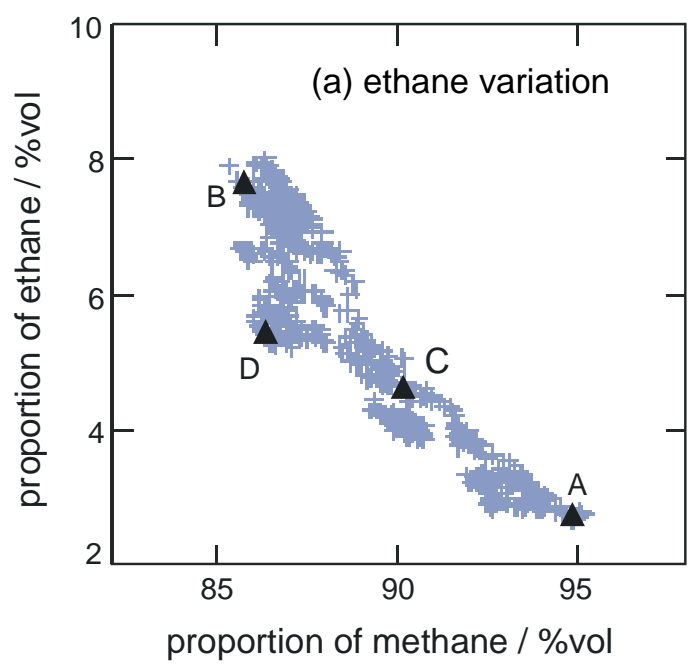

+++ UK sampled data

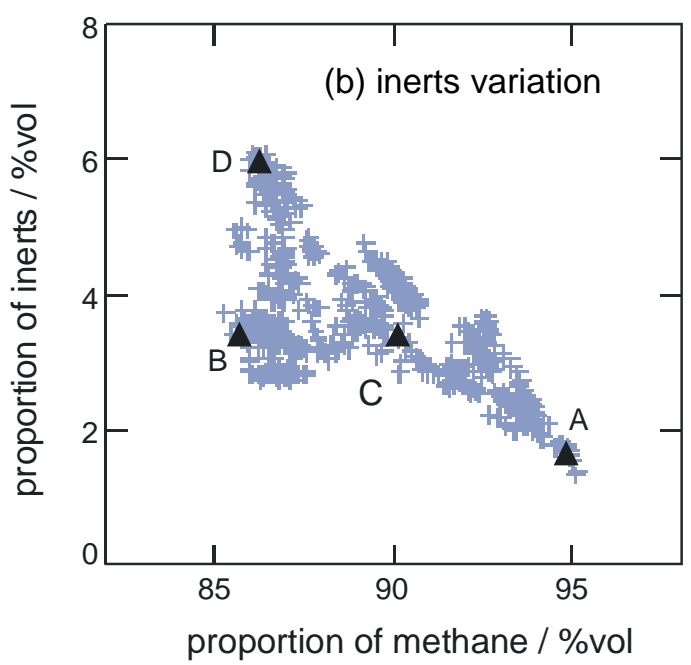

$\mathbf{\Delta} \mathbf{\Delta}$ test compositions

Figure 2. Proportions of ethane and total inerts $\left(\mathrm{CO}_{2}\right.$ plus $\left.\mathrm{N}_{2}\right)$ versus methane proportion, for test gas compositions and the UK composition data used elsewhere in this paper.

\subsection{Performance of pellistor based instrument}

A commercial pellistor based instrument, the GMI Gascoseeker, was tested using the gas mixtures described in section 4.1. The instrument was tested on the \%LEL range, this being arguably the most important for safety reasons and the most difficult for alternative, optical technologies to achieve good performance. It was calibrated using the GMI's automatic gas delivery and calibration system immediately prior to testing. Although the calibration procedure used methane, the Gascoseeker used a "natural gas" calibration setting during measurement, designed to take account of the average methane deficit of natural gas by scaling the raw measurements by a factor of 1.1 before displaying the results.

Each of the gas mixtures was blended with hydrocarbon free air to a concentration of $2.5 \%$ vol (approximately $50 \%$ LEL) by the cylinder supplier (Scott Specialty Gases) and provided with a certificate of analysis.

The measurement errors are shown in Table 4. We need to consider the error range or worst case error rather than the average error here, since instruments should be capable of operation across the whole range of gas compositions that they could reasonably encounter, and because we have no evidence for the distribution of compositional variation. 
Table 4. Measurement errors for a conventional pellistor based instrument (GMI Gascoseeker) when estimating the \%LELs of different synthetic natural gases in the region of 50\%LEL.

\begin{tabular}{|l|c|c|c|}
\hline Mixture & Calculated \%LEL & $\underline{\text { Reading / \%LEL }}$ & $\frac{\text { Proportional error / }}{\underline{\%}}$ \\
\hline Methane control & $51.6 \pm 0.7$ & 52.0 & 0.1 \\
\hline A & $51.9 \pm 0.7$ & 50.0 & -3.7 \\
\hline B & $55.0 \pm 0.7$ & 51.0 & -7.3 \\
\hline C & $53.0 \pm 0.7$ & 50.0 & -5.7 \\
\hline D & $52.4 \pm 0.7$ & 49.0 & -6.5 \\
\hline \multicolumn{2}{|c|}{ Proportional error range (including 100\% methane) / \% } & +0.1 to -7.3 \\
\hline \multicolumn{2}{|l|}{ Proportional error range (natural gas mixtures A-D only) / \% } & -3.7 to -7.3 \\
\hline
\end{tabular}

The performance achieved here is a combination of the normal instrumental repeatability error that might be observed for, say, methane-only measurements, as well as the composition-related uncertainty. Although the instrument was designed to measure natural gas, which should have resulted in it being rescaled to account for the average methane deficit, a systematic error remained. As we are interested here in the error due to compositional variation, there is potential to improve the results by further rescaling, to give a best case benchmark performance proportional error of $\pm 2 \%$ resulting from natural gas variation alone. This is small compared with the normal instrumental error for a single measurement $( \pm 4 \%)$. As there is little point in considering errors below this level, these tests point to a benchmark allowable proportional error of $\pm 4 \%$ on the $\%$ LEL range.

\section{$5 \quad$ Calculated performance of proposed gas - specific detectors}

We took a series of gas compositions and calculated the measurement error that would result from making methane-specific measurements, on the \%volume, \%LEL and ppm scales. For this pseudo Monte Carlo technique, the range of resulting errors was of interest rather than the standard deviation. The technique is appropriate to this case for a number of reasons. Firstly, although the range of proportions of each gas component in natural gas is well known, the concentrations of the components are correlated via the characteristics of different gas fields and via the network entry quality criteria or interchangeability rules that govern the full range of allowed compositions in the network $^{[23]}$. Secondly, we had no information concerning the relative distribution of gas 
compositions following uncertain degrees of downstream mixing in the network, and therefore could not estimate the likelihood of encountering any one composition. In any case, gas detectors would be required to make correct measurements for the full range of acceptable gas compositions in the network. Finally, the method was simple to use, and should be representative of the extremes of what would be encountered in practice, given a sufficiently long and well-chosen list of real compositions.

Basil and Jamieson ${ }^{[24]}$ have used a similar approach to calculating uncertainties in gas metering systems. The method successfully modelled measurement errors in complex systems, and was found to compare well with traditional methods of combining individual measurement errors that arise in different parts of a system.

Here, expected errors were calculated for hypothetical detectors, such as those based on highresolution infrared spectroscopy, capable of making component-specific measurements of methane and / or ethane. It was assumed that our hypothetical instruments were able to make perfect measurements; real instrumental errors would add further uncertainty to a measurement. For \%LEL measurements, the true LEL of each gas mixture was calculated using the method established by Coward and Jones ${ }^{[16]}$, as implemented in the commercial software GasVLe ${ }^{[25]}$. The hypothetical instrument reading, based on the methane and / or ethane content, was compared with this figure to give the proportional error.

For proposed methane - only detectors, we simply took the methane proportion in the gas mixture as the concentration that the instrument would report, and calculated \%LEL concentrations using the LEL figure in Table 1. Where methane plus ethane detectors have been postulated, a two-component approximation to a full natural gas mixture was used as shown below.

On the ppm and \%volume scale, $\quad C_{m i x}=C_{1}+C_{2}$

On the \%LEL scale, $\quad \% L E L_{m i x}=\frac{C_{1}}{L E L_{1}}+\frac{C_{2}}{L E L_{2}}$

$\mathrm{LEL}_{1}$ and $\mathrm{LEL}_{2}$ are the LELs of the individual gases in units of \%vol, whereas $\mathrm{C}_{1}$ and $\mathrm{C}_{2}$ are the concentrations of these components, also in units of \%vol. Equation (3) has been postulated for simple two-component mixtures by Coward and Jones ${ }^{[16]}$. 


\subsection{Errors in measurements of concentration on the ppm and \% vol scales}

On the ppm and \%volume scales, measurements are made in direct proportion to the concentration of gas in air by volume. The errors associated with a perfect methane-specific gas detector will therefore be a direct consequence of the methane deficit in natural gas, ie that fraction of natural gas that does not comprise methane. Figure 3 shows the resulting errors as a function of the proportion of methane in the natural gas mixture, for UK, GERG, US and rest of world (RoW) gas compositions. As expected, the straight line illustrates the direct relationship between the underestimate in concentration and the proportional lack of methane in the natural gas.
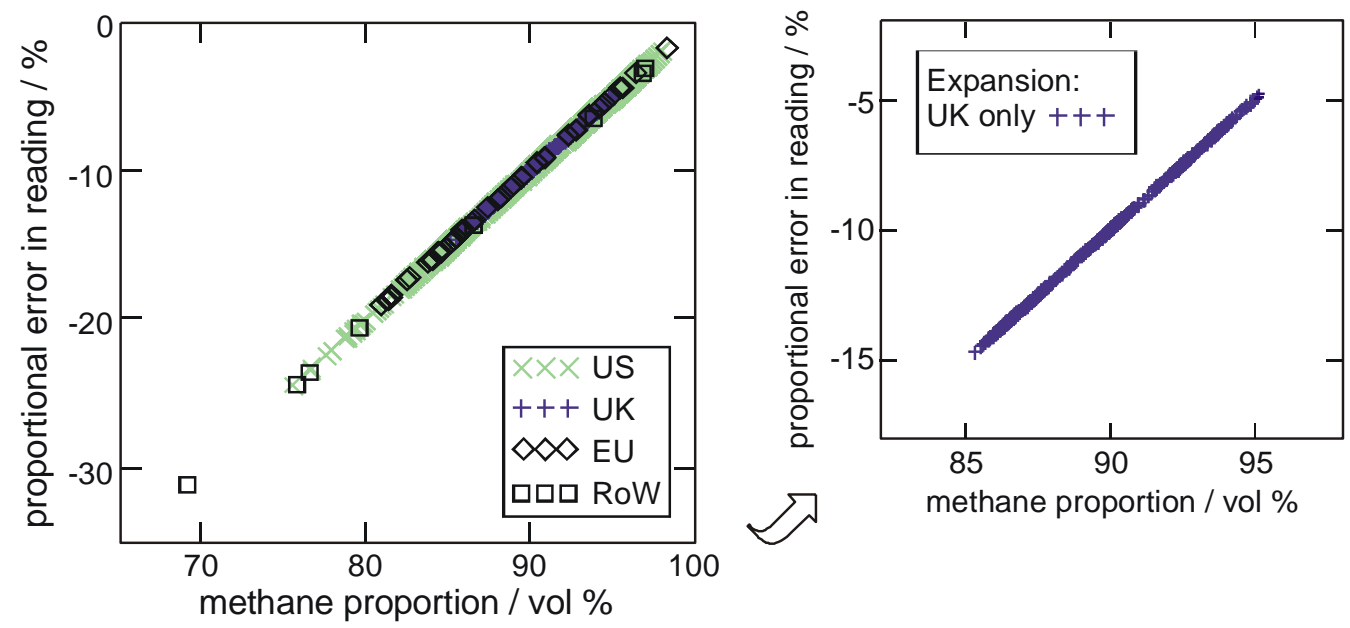

Figure 3. Spread of calculated errors in methane-only based measurements of natural gas on the ppm or \%vol scales, versus the proportion of methane in the natural gas, for various gas compositions. A straight line through the origin follows directly from the mathematical analysis.

For the worldwide gas compositions (including US, EU and RoW), the error would lie between -2\% and $-31 \%$ of the true gas concentration, and likewise between $-5 \%$ and $-15 \%$ for UK compositions. The systematic portion of the error could be removed by either calibrating instruments with a "standardized" natural gas mixture, or by calibrating with methane and then multiplying the instrument reading by a small factor. This normalization would reduce the central error to zero and leave a variable error element of approximately $\pm 18 \%$ (world) and $\pm 5.5 \%$ (UK).

On the ppm scale, such error levels should not present problems in locating leaks, and would be within error limits for other variables as defined in Table 2, so the use of a methane - specific detector could be acceptable in this application. For \%volume scales (see Table 2) the errors are just compatible with IEC/EN gas detector standards (worldwide gases) and with NG standards (UK 
gases), but it should be noted that there would be little headroom to allow for further instrument errors.

Supplementing the methane - only measurement with an additional measurement of the concentration of ethane, according to equation (2), reduces the proportional errors. Figure 4 illustrates the non-normalized errors, which would lie in the range 0 to $-23 \%$ (world) and -2 to $-9 \%$ (UK). If normalized to give zero central error, they would be reduced to $\pm 12 \%$ (world) and $\pm 3.5 \%$ (UK).

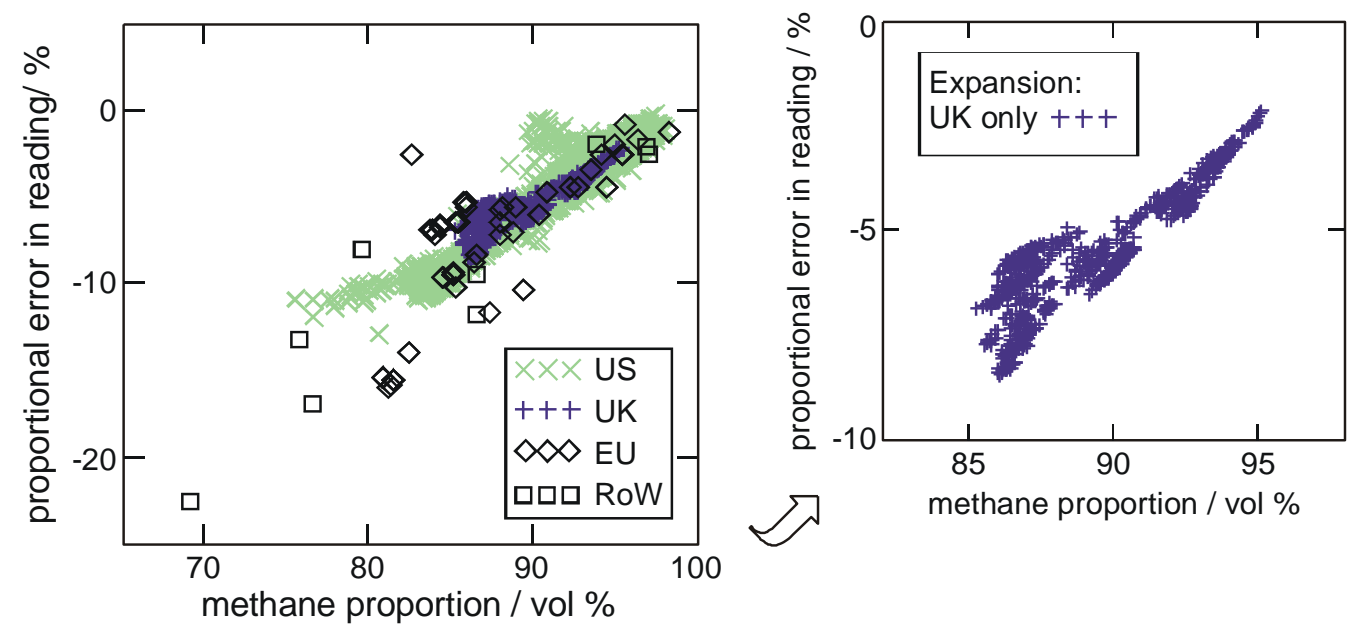

Figure 4. Spread of calculated errors in methane plus ethane based measurements of natural gas on the ppm or \%vol scales, versus the proportion of methane in the natural gas, for various gas compositions.

\subsection{Errors in measurements of concentration on the \%LEL scale}

A measurement on the \%LEL scale indicates how close the gas concentration is to forming a potentially explosive mixture in air. Taking methane as an example, a concentration of $4.9 \%$ volume is equivalent to $100 \%$ LEL (see Table 1). Thus, a true measurement on the LEL scale depends not only on the amount of gas present in air, but also on the concentration at which that gas mixture is at its LEL.

Calculation of LELs of given gas compositions is implemented in the software GasVLe ${ }^{[25]}$, and Figure 5 illustrates the range of LELs calculated for the gas compositions used in this study. 

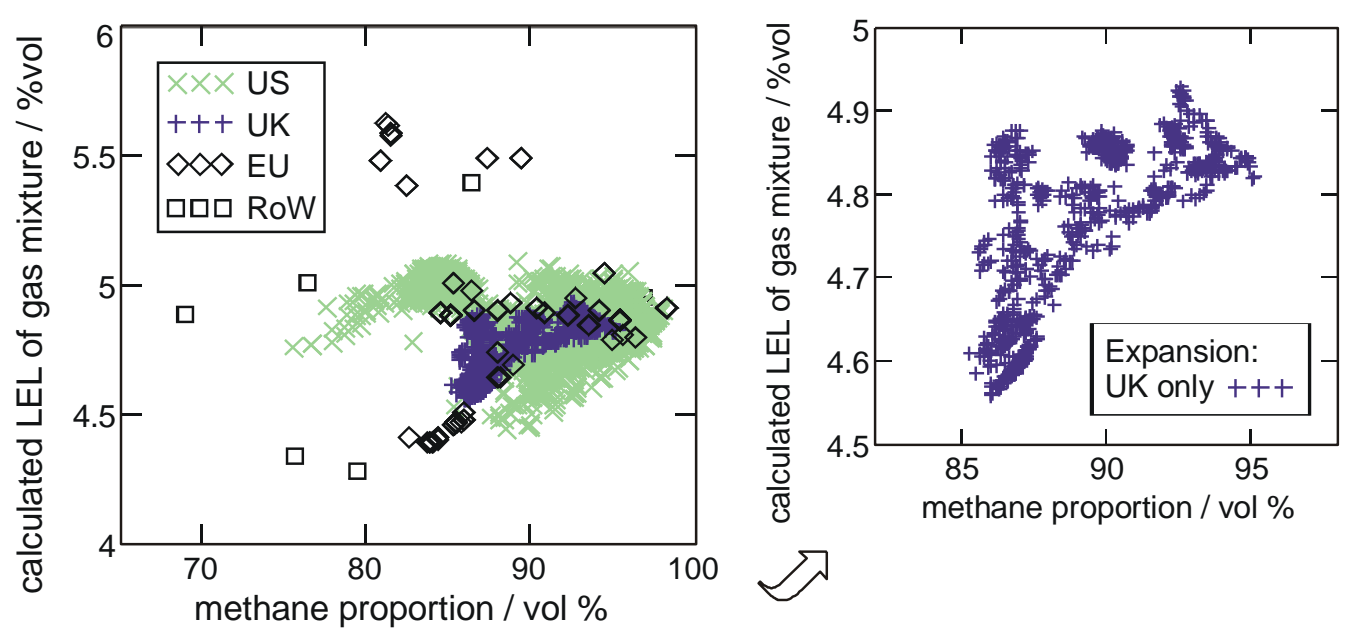

Figure 5. Illustration of the range of calculated true LELs for the gas mixtures used in this study, plotted as a function of the proportion of methane in the mixture.

Using a hypothetical methane-specific measurement of gas concentration on the \%LEL scale results in a range of proportional errors for different gas compositions, as shown in Figure 6. For worldwide compositions the error range is from 0 to $-33 \%$, whereas for UK gases alone the range is from $-6 \%$ to $-20 \%$ LEL. Renormalizing to zero central error gives ranges of $\pm 20 \%$ of the reading (worldwide) or $\pm 8 \%$ of reading $(\mathrm{UK})$.
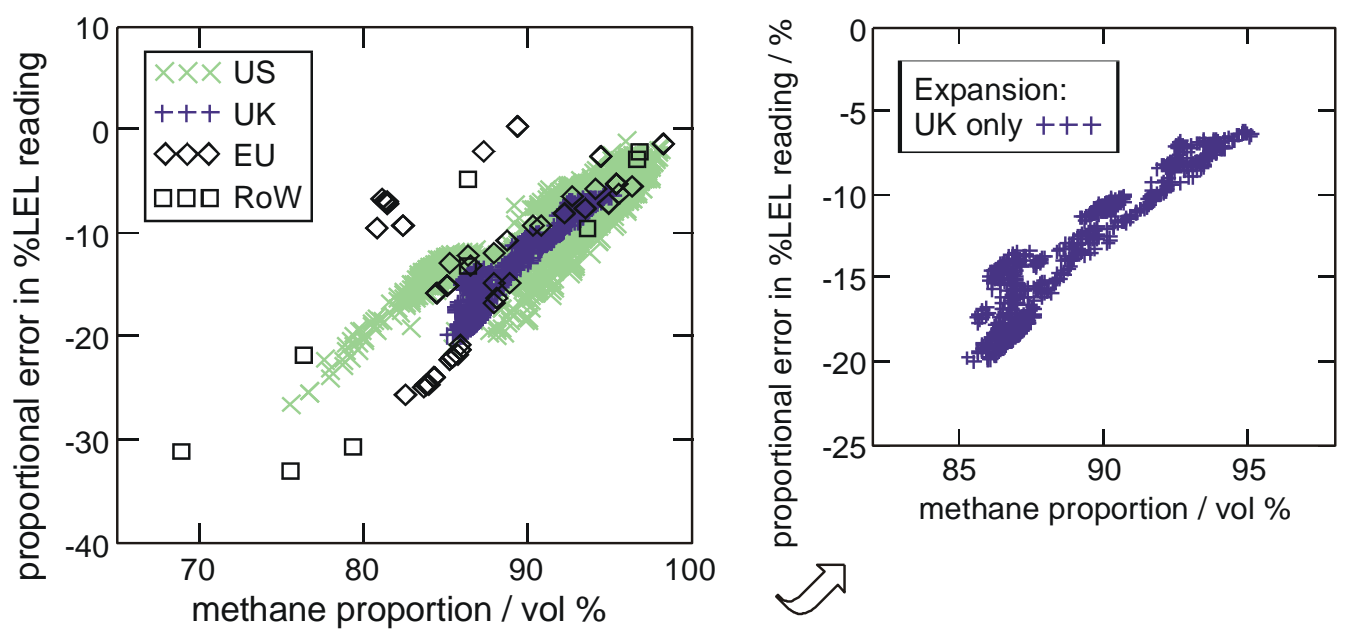

Figure 6. Calculated proportional error in the \%LEL 6of gas leaks measured using a methanespecific detector, versus the proportion of methane in the gas.

An error in the reading from a methane - specific instrument could arise from two possible sources.

i) Methane does not make up $100 \%$ of the gas mixture, so the reading will be an underestimate in proportion to the methane deficit, as was the case for ppm and \%vol scale measurements. 
ii) Variable quantities of other components are present, able to affect the LEL of the mixture. Small levels of higher hydrocarbons such as ethane and propane would lower the LEL of the natural gas mixture such that a methane - only measurement would underestimate the concentration in \%LEL. A high level of inert gases would act to raise the LEL of the gas mixture and could result in an overestimate in the leak concentration in \%LEL.

These two effects can combine to increase the level of uncertainty in the measurement.

As described previously, it would be possible to make a combined methane plus ethane based measurement, using equation (3). The resulting spread of errors is shown in Figure 7 for worldwide and UK gas compositions.
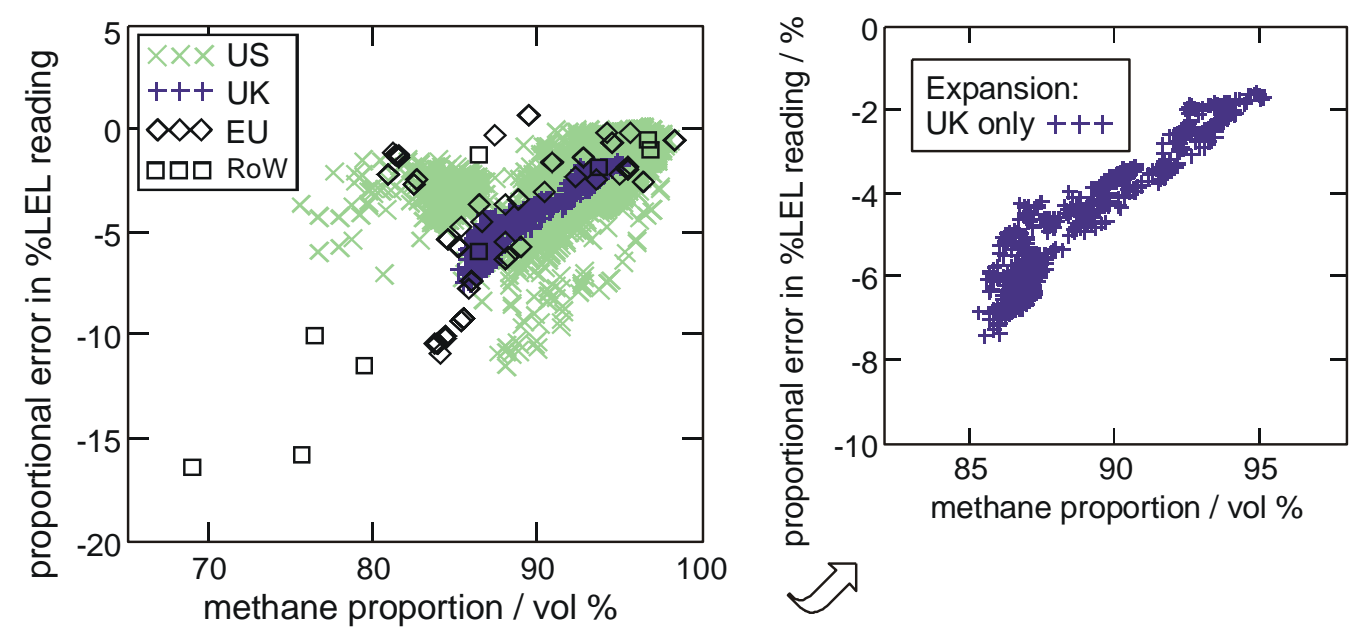

Figure 7. Use of a combined methane plus ethane detector has reduced the spread of calculated proportional errors in the \%LEL reading.

The figures illustrate reduced error levels for an idealized, hypothetical gas detector. Proportional errors lie in the range $+0.5 \%$ to $-16 \%$ of the reading (worldwide) and $-1.5 \%$ to $-7 \%$ (UK). Renormalizing the data gives ranges of $\pm 9 \%$ of the reading (world) and $\pm 3 \%$ of the reading (UK). This is a significant improvement on methane - only calculations and compares well with the benchmark error levels in section 3.

\section{Summary of performance benchmarks and calculations}

We have considered two possible approaches to benchmarks:

(i) To accept a level of performance consistent with established instruments, giving a more stringent performance criterion of around $\pm 4 \%$ of the reading on the \%LEL scale. It would 
have the advantage of not producing a greater level of total error, when faced with a combination of changing instrument repeatability, environmental factors and compositional change, than is presently the case.

(ii) To choose an acceptable error consistent with that allowed for other environmental factors such as temperature or pressure changes. This would categorize compositional change as simply another factor about which neither the instrument nor the operator has any information or control.

Selected benchmark figures, taken from Table 2 and Section 4, are summarized in Table 5 together with the results of calculations from the previous sections.

Table 5. Summary of calculated hypothetical and benchmark error levels for different natural gas concentration measurements. All error ranges shown here are proportional errors in the instrument reading, and assume that instruments can be adjusted to remove the average, systematic error.

\begin{tabular}{|c|c|c|c|c|c|}
\hline $\begin{array}{l}\text { Proposed } \\
\text { detection method }\end{array}$ & $\begin{array}{c}\underline{\mathrm{UK}} \\
\underline{\text { benchmark }}\end{array}$ & $\begin{array}{c}\underline{\text { UK error }} \\
\text { calculations }\end{array}$ & $\begin{array}{l}\text { Worldwide } \\
\text { benchmark }\end{array}$ & $\frac{\text { Worldwide error }}{\underline{\text { calculations }}}$ & Comment \\
\hline \multicolumn{6}{|l|}{ ppm scale } \\
\hline methane - only & \multirow{2}{*}{ $\pm 25 \%$} & $\pm 5.5 \%$ & \multirow{2}{*}{$\begin{array}{c}\text { none } \\
\text { specified }\end{array}$} & $\pm 18 \%$ & \multirow{2}{*}{$\begin{array}{l}\text { methane-only } \\
\text { will suffice }\end{array}$} \\
\hline methane + ethane & & $\pm 3.5 \%$ & & $\pm 12 \%$ & \\
\hline \multicolumn{6}{|l|}{ \%LEL scale } \\
\hline methane-only & \multirow{2}{*}{$\begin{array}{c} \pm 6 \%^{\mathrm{a}} \text { or } \\
\pm 4 \%^{\mathrm{b}}\end{array}$} & $\pm 8 \%$ & \multirow{2}{*}{ $\pm 15 \%$} & $\pm 20 \%$ & \multirow{2}{*}{$\begin{array}{c}\text { methane +ethane } \\
\text { required }\end{array}$} \\
\hline methane + ethane & & $\pm 3 \%$ & & $\pm 9 \%$ & \\
\hline \multicolumn{6}{|l|}{$\%$ vol scale } \\
\hline methane-only & \multirow{2}{*}{ $\pm 6 \%$} & $\pm 5.5 \%$ & \multirow{2}{*}{ $\pm 15 \%$} & $\pm 18 \%$ & \multirow{2}{*}{$\begin{array}{c}\text { methane +ethane } \\
\text { desirable }\end{array}$} \\
\hline methane + ethane & & $\pm 3.5 \%$ & & $\pm 12 \%$ & \\
\hline
\end{tabular}

a Benchmark error from NG standard INQ3; see Table 2 (b).

b Benchmark error based on instruments in current used; see section 4.2.

Note the potential for calculated figures to be in error by up to $\pm 1.5 \%$ (see section 2.1 ).

Systematic underestimates in the measurement of gas concentration are not a fundamental problem, because we are able to predict and correct the level of underestimate within any given gas supply region. Two approaches are possible; (i) calibrate with a "standardized" natural gas instead of pure methane in air, or (ii) multiply the instrument response in software by a correction factor. It has been 
assumed in our calculations that the readings on each scale can be renormalized to remove the average systematic composition-related error and leave only the unpredictable variability.

Errors in ppm measurements are low, and the requirements for this application are not stringent, so a methane-specific detector should be suitable. This is fortunate because ethane would be difficult to detect on this scale, since it makes up only a small proportion of natural gas, which would already be considerably diluted. Sensor technologies for ethane that could be readily incorporated into a lowcost, hand held gas leak detector are currently not available for low ppm measurements.

For \%LEL estimates, the situation is worsened by the fact that compositional variation affects the LEL of the gas as well as the ability of the instrument to detect it. The requirements for instruments on this scale are very stringent, since these measurements play an important role in safety-critical decisions. Methane-specific measurements show fundamental errors that are larger than we would ideally accept. Adding a simultaneous ethane measurement gives a significant improvement, reducing measurement errors to an acceptable level and enabling any instrument to cope with the present degree of variability of composition in gas supplies.

Anticipated error levels are the same for the ppm and \%volume scales, but the requirements for $\%$ volume measurements are more stringent. Comparing the two in Table 5, we may conclude on this scale that methane-only measurements give rise to errors that may be on the margins of acceptability. These uncertainties give little headroom to allow for further instrument errors to creep in, and an additional ethane measurement is perhaps desirable.

\section{Experimental demonstration}

Experiments were performed to assess the performance of gas-specific measurements of methane plus ethane made through tunable diode laser absorption spectroscopy (TDLAS), in comparison with the pellistor based instrument described in section 4.2. Both techniques were assessed on the \%LEL scale with the test mixtures given in Table 3, considered representative of natural gas compositions encountered in the UK.

We are grateful for the loan of a TDLAS based prototype gas detector, which has been previously described $^{[10]}$, from its developers Oliver Hennig and co-workers at Siemens ZT, Munich. The instrument made independent and gas-specific measurements of methane and ethane and contained an implementation of the 2-component approximation to the \%LEL scale according to equation (3). 
It was tested alongside the GMI Gascoseeker in the same series of experiments as described in section 4.2. The results are shown in Table 6 and are directly comparable with those in Table 4.

Table 6. Measurement errors for a prototype TDLAS based instrument, using methane plus ethane measurements, when estimating the \%LELs of with different synthetic natural gases in the region of $50 \%$ LEL.

\begin{tabular}{|l|c|c|c|}
\hline Mixture & True \%LEL & reading / \%LEL & Proportional error / \% \\
\hline Methane control & $51.6 \pm 0.7$ & 53.0 & 2.7 \\
\hline A & $51.9 \pm 0.7$ & 51.0 & -1.7 \\
\hline B & $55.0 \pm 0.7$ & 51.0 & -7.3 \\
\hline C & $53.0 \pm 0.7$ & 49.0 & -7.5 \\
\hline D & $52.4 \pm 0.7$ & 50.0 & -4.6 \\
\hline \multicolumn{2}{|c|}{$\begin{array}{r}\text { Proportional error range (including } \\
100 \% \text { methane) / \% }\end{array}$} & +2.7 to -7.5 \\
\hline \multicolumn{2}{|c|}{$\begin{array}{r}\text { Proportional error range (natural gas } \\
\text { mixtures A-D only) / \% }\end{array}$} & \multicolumn{2}{|c|}{-1.7 to -7.5 } \\
\hline
\end{tabular}

The worst proportional error for the prototype was $-7.5 \%$, which compares favourably with the worst error for the Gascoseeker of $-7.3 \%$. There is potential to reduce the central error to zero to give an error range of $\pm 3 \%$ for natural gas compositional variation alone, with instrument errors adding to the overall level of measurement uncertainty. All these figures are in excellent agreement with the results of calculations in section 5.2. The study therefore provides a practical confirmation of the effectiveness of using independent measurements of methane plus ethane on the most stringent \%LEL scale, with overall performance similar to that of a standard pellistor based instrument.

\section{Conclusions}

This study has considered the effects of the variability of gas composition on a hypothetical gas detector, able to make methane-specific or methane plus ethane based measurements of the concentration of natural gas in air. A practical demonstration of the latter approach has been tested using a prototype instrument based on tunable diode laser absorption spectroscopy.

Variations in the composition of natural gas could give rise to errors in the concentration reading of a methane-specific detector. These errors are fundamental to the application but their extent depends 
on the geographic area within which the instrument will be used. They have been calculated for hypothetical instruments that perform perfectly. Even under these idealized conditions, the range of anticipated errors could be significant; further instrument errors would combine to increase the overall measurement uncertainty. A combined methane plus ethane measuring instrument has been postulated to reduce the errors to an acceptable level, particularly on the \%LEL scale. For any methane- and / or ethane- based measurement of natural gas concentration, a correction should also be made to remove the systematic underestimate in the instrument reading.

Since this issue of gas compositional variation is only partially covered in instrument standards, it has been necessary to use benchmark levels of error in order to decide whether measurement uncertainties would be significant. This has necessarily involved an element of judgement. It is hoped that the results have been presented with sufficient transparency to allow the reader to reach their own conclusions about their significance, and to allow for the use of different benchmarks in regions whose compositions show a different level of variability.

Gas compositional variability has not been a concern for more traditional instruments, but could lead to problems with the acceptance of new technologies. Some optical gas detection instruments are simply designed to respond to methane and are typically tested using methane alone. However, for gas detection applications that demand high accuracy, this study shows that gas sensors should be designed, selected and tested with due consideration to the other components of natural gas.

\section{Acknowledgements}

We are grateful to Oliver Hennig, Rainer Strzoda, Max Fleischer and others at Siemens ZT for the loan of their prototype TDLAS based instrument. This, and the research described in this paper, were developed as part of a collaborative project between Advantica (now GL Noble Denton), Siemens UK and Siemens ZT. We would like to thank Martin Brown of GL Noble Denton for discussions on LELs and for the burning velocity calculations described here. We are also grateful to National Grid for funding our work and for facilitating access to the UK gas composition data used in the study.

\section{References}

[1] IGEM 1998 Dealing with reported gas escapes. Publication SR/20/Edition 2 (The Institution of Gas Engineers and Managers, London) 
[2] Jones E 1987 The pellistor catalytic gas detector, Chapter 2 in Solid State Gas Sensors Moseley PT and Tofield B, Eds (Adam Hilger Series on Sensors)

[3] Fackrell JE 1980 A flame ionisation detector for measuring fluctuating concentration. J. Phys. E: Scientific Instrumentation, 13 888-893.

Simpson C F and Gough T A 1981 Direct quantitative-analysis using flame ionization detection - the construction and performance of the fidoh detector. J. Chromatogr. Sci. 19 $275-82$

[4] Allen MG 1998 Diode laser absorption sensors for gas-dynamic and combustion flows Meas. Sci. Technol. 9 545-562

[5] Werle P 1998 A review of recent advances in semiconductor laser based gas monitors Spectrochim. Acta A 54 197-236

[6] Uehara K, Tai H and Kimura K 1997 Real-time monitoring of environmental methane and other gases with semiconductor lasers: a review Sensor. Actuat. B-Chem. 38-39 136-140

[7] Whitenett G, Stewart G, Atherton K, Culshaw B and Johnstone W 2003 Optical fibre instrumentation for environmental monitoring applications J. Opt. A: Pure Appl. Opt. 5 S140S145

[8] Smith SD, Vass A, Karpushko F, Hardaway H and Crowder JG 2001 The prospects of LEDs, diode detectors and negative luminescence in infrared sensing of gases and spectroscopy Phil. Trans. R. Soc. Lond. A 359 621-634

[9] Pride R D, Hodgkinson J, Murray G, Sutton S and Strzoda R 2001 A new portable gas detection instrument based on a novel optical technique Proc International Gas Research conference (IGRC), Amsterdam Paper DP04

[10] Hennig O, Strzoda R, Magori E, Chemisky E, Tump C, Fleischer M, Meixner H and Eisele I 2003 Hand-held unit for simultaneous detection of methane and ethane based on NIRabsorption spectroscopy Sensor. Actuat. B-Chem 95 151-156

[11] Wild K 2000 Gas quality measurement: A gas control revolution? Gas Eng. Manage. 4012

[12] Jaeschke M and Humphreys A E 1990 The GERG databank of high accuracy compressibility factor measurements. GERG Technical Monograph 4 (VDI)

[13] Liss WE, Thrasher WH, Steinmetz GF, Chowdiah P and Attari A 1992 Variability of Natural Gas Composition in Select Major Metropolitan Areas of the United States GRI report PB92224617 (Gas Research Institute, Chicago, IL, USA) 
[14] Transco 1990 Range of composition of natural gas British Gas Engineering standard Data Sheet BG/PS/DAT33 (updated edition, National Grid, Warwick, UK)

[15] IEC 60079-20:2000. Electrical apparatus for explosive gas atmospheres Part 20: Data for flammable gases and vapours, relating to the use of electrical apparatus (International Electrotechnical Commission)

[16] Coward HF and Jones GW 1952 Limits of flammability of gases and vapours National Bureau of Mines, Bulletin 503 (US Dept of Interior)

[17] Jones GW 1929. Inflammability of mixed gases. US Bureau of Mines Technical Paper 450.

[18] Kee RJ, Grcar JF, Smooke MD and Miller J A 1985 PREMIX. A Fortran Program for Modeling Steady Laminar One-Dimensional Flames. Sandia National Laboratories Report SAND85-8240.

[19] Smith GP, Golden DM, Frenklach M, Moriarty NW, Eiteneer B, Goldenberg M, Bowman CT, Hanson RK, Song S, Gardiner Jr. WC, Lissianski VV and Qin Z 1999 GRI-Mech 3.0 http://www.me.berkeley.edu/gri_mech/

[20] IEC / EN 60079-29-1:2007. Explosive atmospheres. Part 29-1: Gas detectors - Performance requirements of detectors of flammable gases. (British Standards Institution / International Electrotechnical Commission)

[21] Transco 1995 INQ3 Technical specification for portable gas detectors (National Grid, Warwick, UK)

[22] Transco 1984 British Gas Engineering Standard BGC/PS/INQ4 General specification for portable instruments of high sensitivity to detect natural gas (National Grid, Warwick, UK)

[23] See for example, HSE 1998 A Guide to the Gas Safety (Management) Regulations 1996 (HSE Books, HMSO)

[24] Basil M and Jamieson A 1999 Uncertainty of complex systems by Monte Carlo simulation. Meas. Control 32 16-20

[25] GL Industrial Services 2002 GasVLE product information http://www.gasvle.com/ 